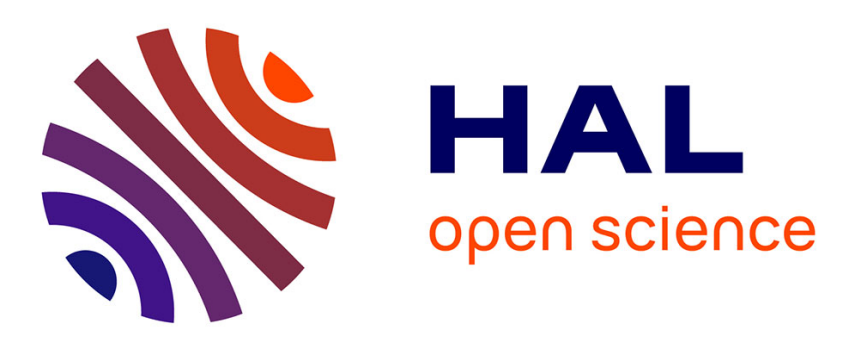

\title{
Simulation of X-ray Traverse Topographs by Means of a Computer
}

\author{
Yves Epelboin, Alain Soyer
}

\section{To cite this version:}

Yves Epelboin, Alain Soyer. Simulation of X-ray Traverse Topographs by Means of a Computer. Acta Crystallographica Section A: Foundations and Advances [2014-..], 1985, 41 (1), pp.67-72. $10.1107 /$ S0108767385000113 . hal-01213427

\section{HAL Id: hal-01213427 https://hal.science/hal-01213427}

Submitted on 3 Nov 2015

HAL is a multi-disciplinary open access archive for the deposit and dissemination of scientific research documents, whether they are published or not. The documents may come from teaching and research institutions in France or abroad, or from public or private research centers.
L'archive ouverte pluridisciplinaire HAL, est destinée au dépôt et à la diffusion de documents scientifiques de niveau recherche, publiés ou non, émanant des établissements d'enseignement et de recherche français ou étrangers, des laboratoires publics ou privés. 
Acta Cryst. (1985). A41, 67-72

\title{
Simulation of X-ray Traverse Topographs by Means of a Computer
}

\author{
By Y. Epelboin AND A. SOYer \\ Laboratoire de Minéralogie Cristallographie, associé au CNRS, Université P et M Curie, \\ 75230 Paris CEDEX 05, France
}

(Received 26 October 1983; accepted 2 August 1984)

\begin{abstract}
The precision of the numerical algorithm used is the critical factor in computing traverse topographs. The varying-step algorithm allows such a computation. Images have been computed simulating the real experiment, i.e. by the addition of the intensities of individual section topographs. Results are in good agreement with experiments: it has been possible to characterize dislocations fully by the study of the fine details of their contrast. This may be of practical interest whenever section topographs cannot be used, as, for instance, in the case of thin crystals.
\end{abstract}

\section{Introduction}

Traverse topography is the most popular topographic method since it permits, in one single experiment, the study of a large volume of a crystal. However, in most cases, one is restricted to qualitative studies. Section topography allows quantitative measurements but its use is limited: the experiment is rather delicate to perform and becomes impossible in the case of thin crystals where the width of the image is too narrow to see any details.

Authier (1967) attempted to explain the contrast of a traverse topograph as the integration, during the scanning of the crystal, of the direct image of the defect. Applying the kinematical theory he could predict the width of the image. This simplified approach is rather satisfactory (Miltat \& Bowen, 1975) but does not permit a full characterisation of the defect.

Quantitative measurements are achieved by the study of the fine details of the contrast of the defect

(C) 1985 International Union of Crystallography 
and simulation has proved to be very efficient in the case of section topography. It would be useful to make the same kind of study in the case of traverse topography. A first attempt has been made by Epelboin (1977). It failed because the precision of the numerical algorithm was not good enough. Petrashen, Chukovskii \& Shulpina (1980) computed an intensity profile in a plane of incidence in the case of a dislocation. They applied the reciprocity theorem (Kato, 1968) to save computation time. However, as will be explained, our tests are not in agreement with their results.

Another conclusion was that simulation of traverse topographs took much too long a time to make it a practical tool for the everyday study of defects.

The appearance of fast specialized computers and the development of fast numerical algorithms for integrating Takagi-Taupin (TT) equations (Takagi, 1962; Taupin, 1964) now permit such simulations.

In this paper we will discuss the basic principles needed to compute traverse topographs. We will then give a brief explanation of a simulation program that has been written to simulate the contrast of straight dislocations. More details about this program will be given elsewhere (Soyer, Epelboin \& Morris, 1984). In the final section of this paper we will show how simulations may help in understanding the contrast of dislocations.

\section{Theory}

\section{Integration method}

Let us consider two kinds of topographic settings:

(a) The source is a point source situated on the entrance surface of the crystal and may be represented as a Dirac function (Kato, 1961).

This is the case of a section topograph. Aristov, Kohn, Polovinkina \& Snigirev (1982) have shown that the non-monochromaticity and the dimensions of the source are the reasons why this approximation is valid. In the real experiment where the incident spherical source possesses a finite lateral extension each point along the surface acts as an incoherent spherical source.

(b) The source is a plane wave of infinite lateral extension.

In these two settings the amplitude distributions are Fourier transforms. Thus, applying Parseval's theorem, the integrated intensity, i.e. the intensity distributions, in a traverse topograph is identical.

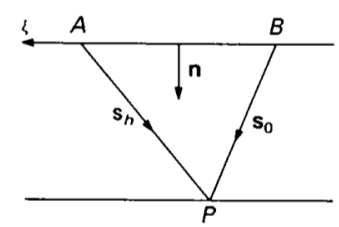

Fig. 1. Definition of the geometry.
The integrated intensity may be written (Takagi, 1969):

$$
I_{h}(P)=\int_{B}^{A}\left|U_{h}(P, \xi)\right|^{2}\left|D_{a}(\xi)\right|^{2} K \gamma_{0} \mathrm{~d} \xi,
$$

where $U_{h}(P, \xi)$ is the amplitude of a reflected wave corresponding to a point source located at point $\xi$ on the entrance surface, $D_{a}(\xi)$ is the amplitude of the incident wave, $\gamma_{0}=\cos \left(\mathbf{n}, \mathbf{s}_{0}\right)$ and $K$ is the wave number of the incident wave (Fig. 1).

Thus, in a traverse topograph the intensity at point $P$ is a function of the intensity arriving at this point from the entrance surface. It is completely independent of the intensity distribution along $\xi$ or the shape of the wave front incident on the crystal. The integrated intensity may be calculated in two different ways; the first is to add the intensities of incident plane waves giving to each one a different departure from the exact Bragg condition:

$$
I_{h}(P)=\int_{-\infty}^{+\infty} I_{h}[\delta(\Delta \theta)] \mathrm{d}[\delta(\Delta \theta)]
$$

where $\delta(\Delta \theta)$ is the departure from the exact Bragg angle of the incident plane wave. The second method is to simulate the real experiment, i.e. to move the point source along the entrance surface and to add together the intensities of all individual section topographs (Fig. 2):

$$
I_{h}(P)=\int_{B A} I_{h}(\xi) \mathrm{d} \xi
$$

where $\xi$ is the position of each incident spherical wave along the entrance surface.

The precision of the numerical algorithm used to integrate the TT equations is a very crucial point. Epelboin (1977) has shown that (2) should not be used: as usual in numerical methods the limits of the integral must be replaced by finite values and their choice is rather delicate. The integration step $\mathrm{d}(\delta(\Delta \theta))$ must be small enough, which requires a tremendous number of calculations. Moreover, the numerical errors increase with $\delta(\Delta \theta)$ when computing the intensity for one plane wave of given $\delta(\Delta \theta)$ departure from the exact Bragg condition, so that results are wrong. Using (3), the integration is performed along a limited distance and, as will be explained later, the integration step $d \xi$ is a function of the required resolution only.

\section{Accuracy of the numerical algorithm}

To compute a traverse topograph from $a$ to $b$ (Fig. 2) it is thus necessary to add the intensities of individual section topographs with point sources moving from $O$ to $O^{\prime}$. The results of Epelboin (1977) were not satisfactory because the precision of the numerical algorithm to integrate the TT equations was not good enough. 
Partial derivative equations such as the TT equations may be computed in different ways; we use the half-step derivative method as described by Authier, Malgrange \& Tournarie (1968). The calculation is performed along a set of characteristic lines parallel to the refracted $\mathbf{s}_{0}$ and diffracted $\mathbf{s}_{h}$ directions (Fig. 3). The amplitudes of the waves at point $A$ depend on the amplitudes at points $B$ and $C$ only. Nourtier \& Taupin (1981) have shown that for a given precision this is the best method.

Let us call $p$ and $q$ the elementary steps of integration $A C$ and $B C$ (Fig. 3). The choice of their lengths must be made according to the following conditions:

$p$ and $q$ must be small enough to take into account the oscillations of the waves, especially near the edges of the Borrmann fan.

$p$ and $q$ must be large enough to avoid numerical errors and save computing time, which is proportional to $1 / p^{2}$ and $1 / q^{2}$.

The first point is of special importance when simulating traverse topographs and explains the failure (Epelboin, 1977) of algorithms in which the integration steps are constant (CSA). Petrashen (1976) and Epelboin (1981) have suggested using a varying-step algorithm (VSA) in which $p$ and $q$ may become small enough to follow the rapid oscillations of the waves near the edges of the Borrmann fan. Fig. 4 is a schematic representation of the VSA as described by Epelboin (1983).

One of the interests of VSA is to be able to adapt the local steps to the local variations of the amplitudes of the wave fields before any integration. Since analytical solutions exist for a perfect crystal it is possible to choose the steps of integration by computing the positions of the extinction fringes inside a perfect crystal. The steps $p$ and $q$ are small near the edges of the Borrmann fan and increase in the middle of the triangle where the waves vary slowly. In a deformed crystal the network of integration is modified where a defect crosses the refracted

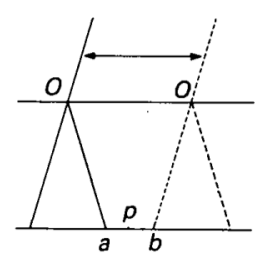

Fig. 2. Principle of the simulation of traverse topographs.

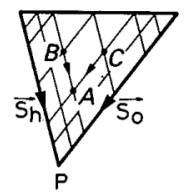

Fig. 3. Principle of numerical integration. direction $\mathbf{s}_{0}$ and the density of nodes is increased along the $s_{h}$ direction (Fig. 4).

One advantage of the VSA is that it is more accurate than a CSA and at the same time faster because the integration steps are always adapted to the local variations of the wave amplitudes.

\section{Criticism of the use of the reciprocity theorem}

The basic principle of computing a traverse topograph is very simple: according to (3) one simulates individual section topographs corresponding to a discrete distribution of sources between $B$ and $A$ (Fig. 1 ) and adds their contributions at point $P$. Their number depends on the width $a b$ (Fig. 2) of the simulation and also on the resolution of the calculation. Let $\Delta$ be the distance between two successive points along the exit surface (Fig. 5). The distribution of sources along $A B$ must be equal to the distribution of points $P$ along $a b$. This requires a tremendous number of calculations since $\Delta$ may be as small as $0.2 \mu \mathrm{m}$ and means an unrealistic computation time.

To avoid this difficulty Petrashen et al. (1980) suggested using the reciprocity theorem. Setting a point source at $P$ along the exist surface, Kato (1968) has shown that

$$
I_{h}(P)=\int_{-A}^{B}\left|U_{h}(\xi, P)\right|^{2} \mathrm{~d} \xi
$$

where $U_{h}(\xi, P)$ is now the amplitude of the reflected wave travelling from $P$ to any point between $A$ and $B$ along the entrance surface. There is no longer any correlation between the distributions of points along $a b$ and $\xi$. Along the exit surface the distance between

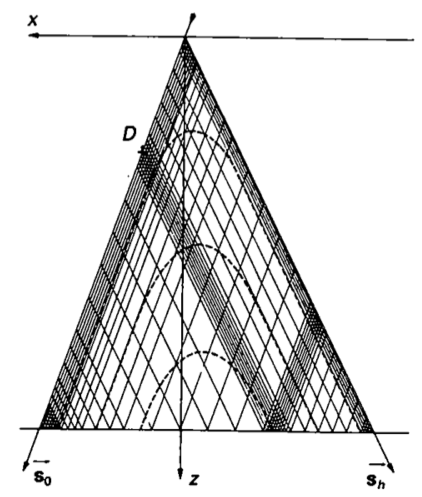

Fig. 4. Varying-step network of integration.

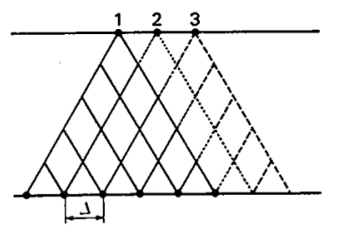

Fig. 5. Addition of elementary section topographs. 
two computed points $P$ depends only on the resolution necessary for the simulation and not on the density of nodes in the network of integration of the individual section topographs.

We have computed intensity profiles along $a b$ for a crystal containing a dislocation and for various diffraction conditions using either (3) or (4). The profiles are different, (4) always giving lower values than (3) in the areas corresponding to the direct image of the defect. We suspect that this difference comes from the limited accuracy of the VSA in the most disturbed areas near the core of the defect. It would be necessary to use a more sophisticated numerical method to integrate the TT equations. One method could be to perform the integration twice: a first time using the VSA then from these results to build a new network of integration. Another possibility would be to use a more precise method than the half-step derivative method. But the computation time would be much too long.

The quality of the simulations using (3) shows that the accuracy of the VSA is good enough to compute traverse topographs simulating the real experiment.

\section{Problem of the sampling of the sources}

Using a VSA means that the distance between the points along the exit surface at which the intensity is computed in one section topograph varies (Fig. 4). To draw an image where the pixels are equally spaced, these points must be replaced by a distribution of equally spaced points (Fig. 5) matching the required resolution $\Delta$. These points are computed by interpolation. $\Delta$ is also the integration step of (3). The intensity at a given point $P$, in the traverse topograph, is computed by adding all the intensities from the various section topographs arriving at this point. The higher the required resolution, the greater the number of computed section topographs. Thus, the step $\Delta$ is independent of the values of the integration steps.

\section{A simulation program: $A D E L E$}

The simulation program that we have written will be described in another paper (Soyer et al., 1984). We will briefly give its basic principles.

On an IBM 370/168 computer the calculation of the intensity along the exit surface for a single section topograph in one incident plane needs about $2 \mathrm{~s}$ for a silicon wafer $400 \mu \mathrm{m}$ thick, 220 Mo $K \alpha$ reflection. For a $320 \mu \mathrm{m}$ large image and a resolution of $1.6 \mu \mathrm{m}$ each line of the image is computed in about $600 \mathrm{~s}$. To speed up the calculation we usually compute every third line, the two missing ones being calculated by linear interpolation. A whole $320 \times 320 \mu \mathrm{m}$ image is computed in about $11 \mathrm{~h}$ ! It is thus worthwhile to try to decrease this enormous time as much as possible. This may be achieved by the programming itself and also by using an array processor.

\section{Minimizing the number of elementary calculations}

To compute an image of width $a b$ (Fig. 2) the point source is scanned from $O$ to $O^{\prime}$. It is obvious that near the edges of the image only a small fraction of the nodes in the network of integration contributes to the calculation. For instance, when the point source is at point $O$, it is sufficient to integrate the equations along $O a$ only. The computation may be restricted to the nodes contained in $O O^{\prime} a b$. Since the integration is performed along characteristic lines parallel to $\mathbf{s}_{0}$ (Fig. 4) starting from the refracted direction, it is easy to introduce such limitations.

\section{Using an array processor}

An array processor is a decidated machine attached to a host computer. It performs specialized calculations when requested by the host. The array processor may be started either by the use of specialized Fortran mathematical libraries or by writing programs in its assembler language. It can be attached to a minicomputer and runs at a speed comparable to that of giant computers. This speed is achieved by special hardware designed for scientific calculations.

The simulation program, $A D E L E$, was first written in Fortran. The integration subroutines were then rewritten in APAL (assembler language for FloatingPoint Systems array processors). Our tests have shown that for a given image a line is computed in $6 \mathrm{~h}$ using a minicomputer (Bull Mini6/53 comparable to a DEC $11 / 70$ ), in $40 \mathrm{~min}$ using an IBM $370 / 168$, in 4 min using a Cray 1 with the Fortran version and in 13 min using the FPS AP100 array processor linked to the Mini6/53.

This means that the use of an array processor permits images to be computed on a small local machine.

\section{Study of the contrast of dislocations}

Fig. 6 is the traverse topograph of a straight dislocation in silicon (220 reflection, Mo $K \alpha$ ). It is inclined in the crystal; simulations of section topographs (Epelboin, 1974) show that the positions of the white and black fringes in the intermediary image are reversed by changing the sign of the Burgers vector. Fig. 7 shows the corresponding simulation. Fig. 8 is the simulation of the same dislocation except that the Burgers vector has been reversed from $\frac{1}{2}[101]$ to $\frac{1}{2}[\overline{10} \overline{1}]$.

The output device is a Numelec Pericolor 2000 raster picture system, which produces a high-quality image on a television screen.

We note that a white contrast is more visible in Fig. 7 than in Fig. 8 near the point where the dislocation crosses the exit surface. Hyperbolic black fringes appear in Fig. 8. Their contrast is reversed compared with that of the same ones less visible in Fig. 7: these fringes are due to the interaction of diffracted wave 
fields with normal ones and this phenomenon may be described by analogy with the $I_{3}$ fringes as described by Authier (1968) in the case of a stacking fault. Notice another system of fringes in the left part of the image that is not visible in the real experiment. This is due to the noise of the photographic emulsion and to its limited resolution, which is much better in the simulation. We may conclude that the dislocation in Fig. 6 corresponds to Fig. 7. This is in agreement with the simulations of section topographs.

Fig. 9 shows the image of dislocations in a silicon wafer (333 reflection, Mo $K \alpha$ ) (Lefeld-Sosnowska, 1983). The angle between the faces of the wafer and the defect is small; this explains why the contrast is poor: the intermediary image does not exist. Fig. 10

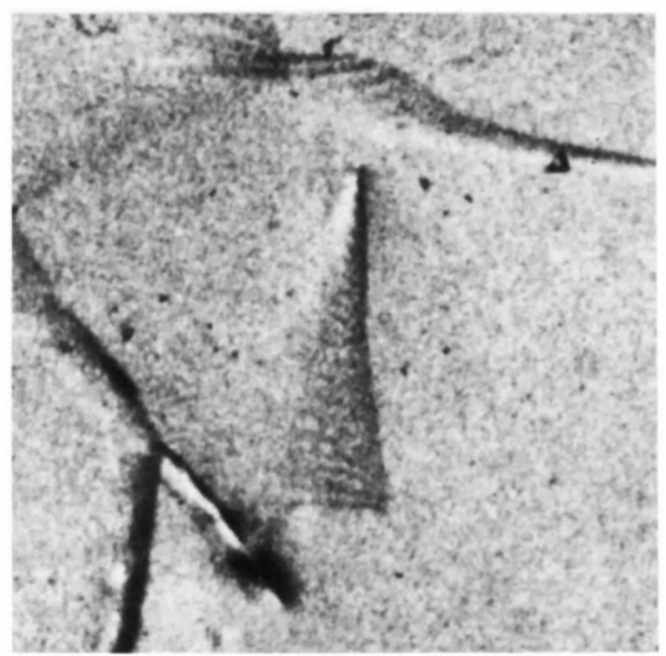

Fig. 6. Traverse topograph, Mo $K \alpha, 220$ reflection, Si, $800 \mu \mathrm{m}$.

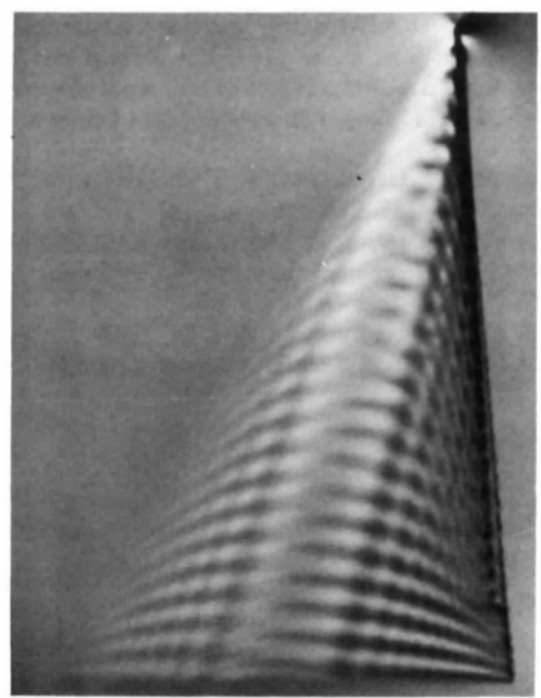

Fig. 7. Simulation of topograph of Fig. $6, b=\frac{1}{2}[101]$. Resolution $0.8 \mu \mathrm{m}$

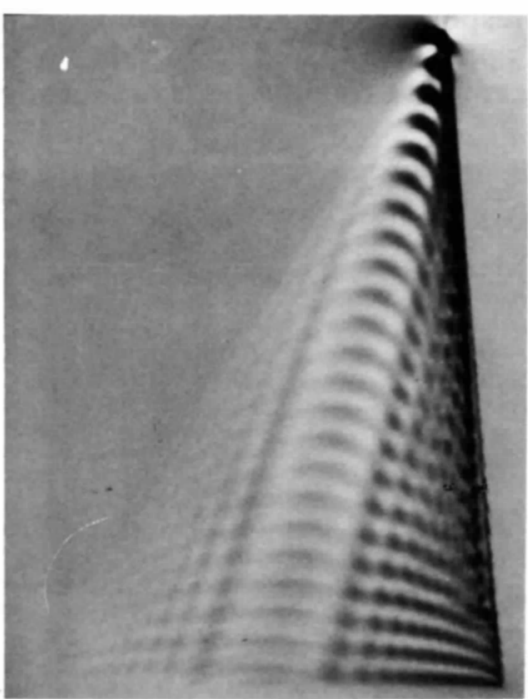

Fig. 8. Simulation of topograph of Fig. $6, \mathbf{b}=\frac{1}{2}[\overline{1} 0 \overline{1}]$.

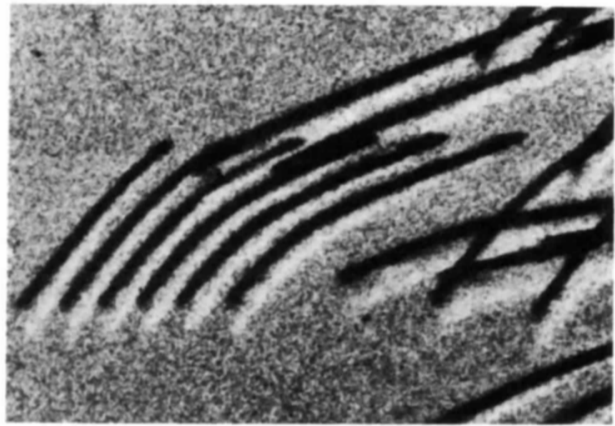

Fig. 9. Traverse topograph, Mo $K \alpha, 333$ reflection, Si $400 \mu \mathrm{m}$.

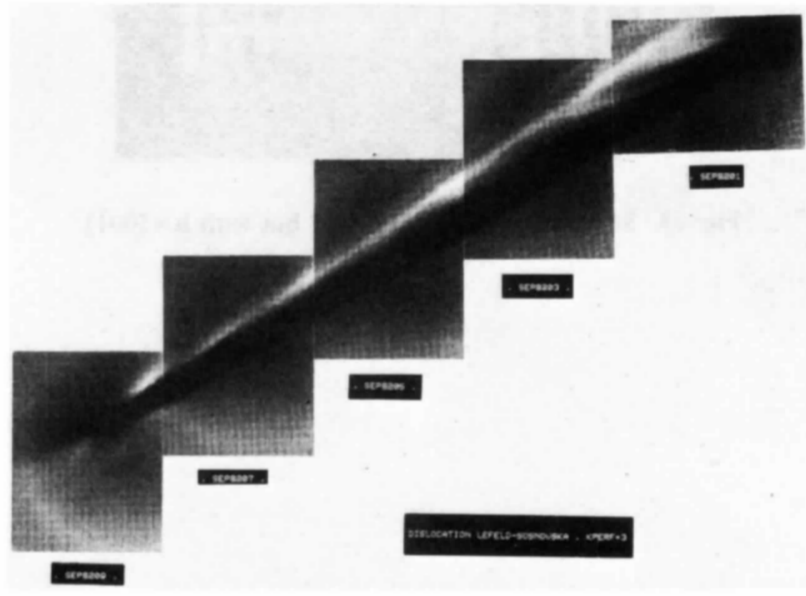

Fig. 10. Simulation of topograph of Fig. 9. Resolution $0 \cdot 8 \mu \mathrm{m}$. 
is the corresponding simulation. We have also simulated the opposite Burgers vector but no significant difference could be found. This is not surprising since

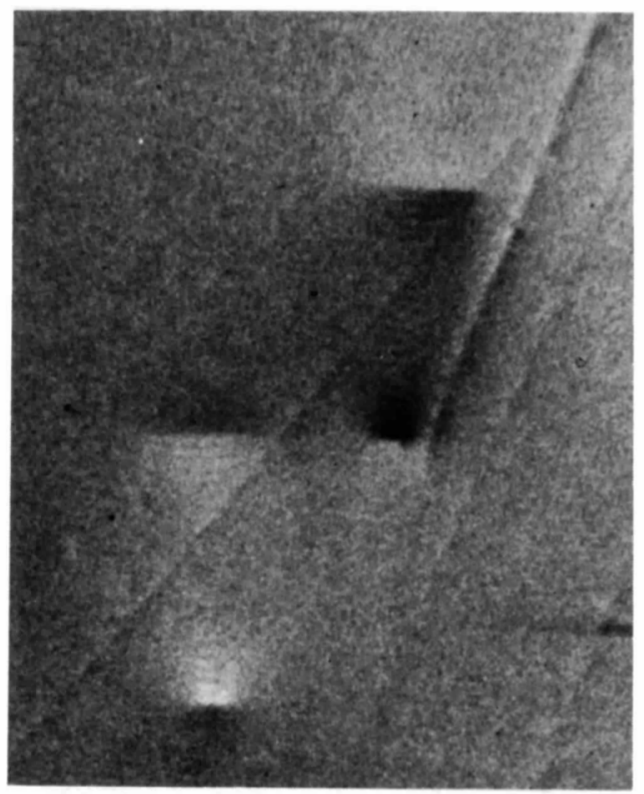

Fig. 11. Traverse topograph, Mo $K \alpha, 200$ reflection, KDP $1100 \mu \mathrm{m}$.

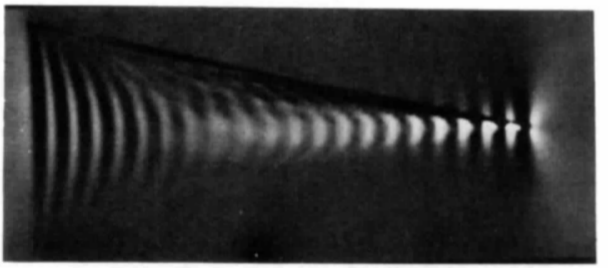

Fig. 12. Corresponding simulation of Fig. 11, $\mathbf{b}=[001]$.

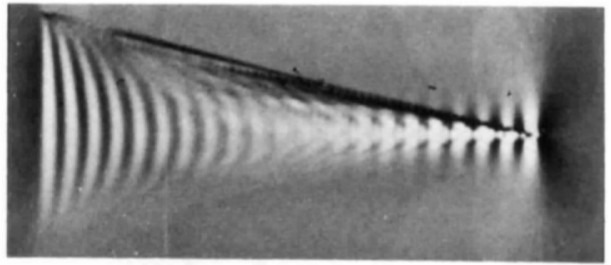

Fig. 13. Same simulation as Fig. 12 but with $\mathbf{b}=[00 \overline{1}]$. it is not possible to identify any characteristic part in this contrast where just a black and white contrast is visible. It would be necessary to make another topograph with a different reflection where the dislocation presents a more detailed image.

Fig. 11 shows dislocations in KDP. They make an angle of about $30^{\circ}$ with the surface of the crystal. The sense of their Burgers vector has been found by study of surface effects (Dunia, Malgrange \& Petroff, 1980) and by simulations of section topographs. Their Burgers vector is [001]. Simulations of Figs. 12 and 13 permit both directions for the Burgers vector to be distinguished: a first black fringe near the entrance surface corresponds to $\mathbf{b}=[00 \overline{1}]$. An additional black direct image appears in the simulations. It might be because of incorrect elastic stiffness values or of a relaxation in the core of the dislocations.

We are greatly indebted to F. Morris whose help was absolutely necessary in programming the array processor.

\section{References}

Aristov, V. V., Kohn, V. G., Polovinkina, V. I. \& Snigirev, A. A. (1982). Phys. Status Solidi, A, 72, 483-491.

Authier, A. (1967). Adv. X-ray Anal. 10, 9-31.

Authier, A. (1968). Phys. Status Solidi, 27, 77-93.

Authier, A., Malgrange, C. \& Tournarie, M. (1968). Acta Cryst. A24, 126-136.

Dunia, E., Malgrange, C. \& Petroff, J. F. (1980). Philos. Mag. A, 41, 291-306.

Epelboin, Y. (1974). J. Appl. Cryst. 7, 372-377.

Epelboin, Y. (1977). Acta Cryst. A33, 758-767.

Epelboin, Y. (1981). Acta Cryst. A37, 132-133.

EPELboin, Y. (1983). Acta Cryst. A39, 761-767.

Kato, N. (1961). Acta Cryst. 14, 627-635.

KATO, N. (1968). Acta Cryst. A24, 157-160.

Lefeld-SOSNOWSKA, M. (1983). Private communication.

Miltat, J. \& Bowen, D. K. (1975). J. Appl. Cryst. 8, 657-669.

Nourtier, C. \& Taupin, D. (1981). J. Appl. Cryst. 14, 432-436.

Petrashen, P. V. (1976). Fiz. Tverd. Tela (Leningrad), 18, 37293731.

Petrashen, P. V., Chukovskit, F. \& Shulpina, I. L. (1980). Acta Cryst. A36, 287-295.

SOYer, A., Epelboin, Y. \& Morris, F. (1984). Submitted to $J$. Appl. Cryst.

TAKAGI, S. (1962). Acta Cryst. 23, 23-25.

TAKagi, S. (1969). J. Phys. Soc. Jpn, 26, 1239-1253.

TAUPIN, D. (1964). Bull. Soc. Fr. Minéral. Cristallogr. 87, 469-511. 